\title{
Symposium review: Host-rumen microbe interactions may be leveraged to improve the productivity of dairy cows ${ }^{1}$
}

\author{
D. M. Bickhart ${ }^{2}$ and P. J. Weimer \\ US Dairy Forage Research Center, Agricultural Research Service, USDA, Madison, WI 53705
}

\begin{abstract}
The rumen is a large bioreactor that enables dairy cattle to derive nutrition from otherwise indigestible plant polymers and compounds. Despite the direct contribution of the rumen's microbial community toward the nutrition of the dairy cow, only a general knowledge has been gained of the metabolic processes within the rumen, and less still is known about most of the individual microbial species that colonize the organ. What has been discovered is that the rumen contains a diverse community of microbial species from all of the major domains of life, and that the contents of the rumen can vary greatly among individual animals. Preliminary evidence also indicates that rumen microbial profiles are heritable and sustainable within an individual, and that rumen microbial community structure can revert to its original profile within a short period following substantial perturbation. Much progress has been made in recent years to identify the diversity of microbial species in the rumen; however, the most popular methods used to identify microbial species often lack the predictive power necessary to associate particular microbial profiles with rumen metabolic activity. This represents the most significant barrier to the design of models that can estimate the direct effects of rumen microbial content on downstream dairy production traits. If such challenges can be overcome, it is possible that rumen microbial content could be assessed as a new phenotypic trait in cattle. In the future, we may estimate dairy production using a "genotype $\times$ environment $\times$ microbial" interaction model that accurately combines all factors affecting milk production.
\end{abstract}

Key words: rumen, bioreactor, host-microbe interaction, microbial community

\footnotetext{
Received June 13, 2017.

Accepted August 31, 2017.

${ }^{1}$ Presented as part of the Ruminant Nutrition Symposium: Ruminal Metagenomics in Dairy Cattle-Beyond Microbial Diversity at the ADSA Annual Meeting in Pittsburgh, Pennsylvania, in June 2017.

${ }^{2}$ Corresponding author: Derek.bickhart@ars.usda.gov
}

\section{IMPORTANCE OF THE MICROBIAL COMMUNITY IN CATTLE RUMINATION AND PRODUCTION}

The evolution of the gastrointestinal tract is a history that has been pockmarked by many innovations and compromises; however, the defining element of this history is the tacit acknowledgment of defeat: colonizing microbes are here to stay. Some mammals - particularly carnivorous species - increase their absorption of nutrients from food by using a shorter gastrointestinal tract and decreasing food transit time to reduce feed energy losses to microbes in their large intestines. Other, primarily herbivorous, species form a symbiosis with microbial colonizers, for which they provide a suitable environment for microbial growth; in return, the microbes provide their host with nutrients from otherwise indigestible food sources. It is in this context that the symbiosis between cattle and microbes becomes apparent, as the rumen is a highly specialized organ designed to contain and promote a collection of mutualistic microbial species while simultaneously harvesting nutrients derived from their digestion of plant fiber and cellular material.

The rumen interior is lined by a stratified squamous epithelium (epimural layer) that changes texture and color in response to mechanical and chemical stimuli (Baldwin et al., 2004). A critical adaptation in the evolution of the rumen was an expansion in its volume, relative to the rest of the gastrointestinal tract. In mature Holstein cows, for example, the rumen volume generally ranges from 70 to $100 \mathrm{~L}$, and the rumen and its contents account for about one-seventh of the animal's total BW. This expansion established a solids retention time long enough to permit microbial degradation of fibrous plant materials to acetate and other VFA. However, this retention time is too short to permit the establishment of slow-growing, thermodynamically constrained microbes capable of VFA degradation such as proton-reducing acetogens (which convert $\mathrm{C}_{3}-\mathrm{C}_{6}$ VFA to $\mathrm{H}_{2}$ and acetate) or aceticlastic methanogens (which cleave acetate to methane and $\mathrm{CO}_{2}$ ). By preventing catabolism of VFA by other members of the ruminal community, up to $75 \%$ of the energy in the original 
Table 1. Major phyla frequently observed in the rumen

\begin{tabular}{|c|c|c|c|c|}
\hline Phylum & Genus & Domain & $\begin{array}{c}\text { Relative } \\
\text { abundance }(\%)^{1}\end{array}$ & Major metabolic activity \\
\hline \multirow[t]{2}{*}{ Bacteroidetes } & & Gram-negative bacteria & $\sim 30$ & Propionate or succinate production \\
\hline & Prevotella & Gram-negative bacteria & $\sim 20$ & Propionate or succinate production \\
\hline \multirow{3}{*}{ Firmicutes } & Butyrivibrio & Gram-positive bacteria & $\sim 4$ & Butyrate production \\
\hline & Lachnospira & Gram-positive bacteria & $\sim 6$ & Pectin fermentation and formate production \\
\hline & Succiniclasticum & Gram-positive bacteria & $\sim 1$ & Succinate consumption, propionate production \\
\hline Euryarchaeota & Methanobrevibacter & Archaea & $\sim 1$ & Methane-producing \\
\hline Protists & & Eukarya & $\sim 40$ & Lipid production, competition with host \\
\hline
\end{tabular}

${ }^{1}$ Relative abundance percentages derived from Henderson et al. (2015) but are highly variable among studies, likely due in part to differences in DNA isolation and analysis methods.

digestible organic matter in the feed is retained within the VFA for absorption from the rumen and intestines, although a large fraction of the VFA is consumed by oxidation in the ruminal epithelial cells (Bergman, 1990). Each VFA is used for a different function, with acetic acid used for ATP and lipid synthesis, propionic acid for gluconeogenesis in the liver, and butyric acid converted immediately into a ketone body by enzymes in the rumen epimural layer for use as an energy source for many tissues of the body. Apart from the conversion of butyric acid in the rumen epimural layer, the majority of enzymatic activity aiding cattle digestion is provided by a diverse group of commensal microbes inhabiting the lumen of the rumen; henceforth called the "rumen microbiome."

The rumen microbiome consists of microbial groups that represent all 3 of Woese's domains of life: bacteria, archaea, and eukarya (Kim et al., 2011). The bacteria comprise thousands of different species and approximately $60 \%$ of the microbial mass, and are primarily responsible for most of the feed biopolymer degradation and fermentations of the resulting monomers and oligomers. This community is dominated by 2 phyla (see Table 1): Firmicutes (particularly represented by the genera Butyrivibrio, Lachnospira, Succiniclasticum, and Ruminococcus) and Bacteroidetes (particularly represented by the genus Prevotella), that together comprise $\sim 90 \%$ of the $16 \mathrm{~S}$ rRNA gene abundance (a proxy for community abundance) in bulk ruminal contents (Henderson et al., 2015). The archaea, which comprise only $\sim 1 \%$ of the microbial mass, are primarily hydrogenotrophic methanogens, mostly of the genus Methanobrevibacter, with additional contribution from methylotrophic methanogens, particularly Methanothermoplasmata (Poulsen et al., 2013). The eukaryotes are primarily represented by various genera of protists ("protozoa") that can comprise one-third or more of the microbial mass, plus anaerobic chytrid fungi that comprise only $\sim 1 \%$ of microbial mass in ruminants from temperate climates but are more prevalent in tropical ruminants. It is important to note that rumen microbial content differs between the 2 different phases of rumen digesta material: the liquid phase and solid phase contain different community compositions (Kong et al., 2010). Of these 2 phases, the solid phase can contain $80 \%$ or more of the bacterial community (Yang et al., 2001) and is likely to contain the most important microbes related to digestion of feed particles.

\section{PHENOTYPING RUMEN MICROBIAL POPULATIONS}

Rumen microbial diversity can be readily assessed through ribotyping experiments to generate relative estimates of species presence/absence and diversity indices. By sequencing and aligning variable portions of highly conserved genes, such as those encoding small subunit (prokaryotic 16S or eukaryotic 18S) ribosomal RNA, one can identify sequence variants that are predictive of different microbial genera, species, and strains. This information is used to identify operational taxonomic units (OTU), which are, in turn, often used (and occasionally misused) as a proxy for predicted metabolic activity. Using these predictions and assessments of relative abundance of microbial species, hypotheses on metabolic activity can be generated. However, the identification of specific variants that account for individual, metabolically important strains is highly subject to sampling bias and may influence downstream functional interpretations (Nguyen et al., 2016). This is best demonstrated by the fact that comprehensive ribotyping experiments find difficulty in assigning sequence reads to individual microbial species, with one such survey even failing to assign $70 \%$ of reads to a defined genus (Henderson et al., 2015). Microbial metabolic capabilities can vary greatly among strains (the lowest, base unit of taxonomic identity) of 
Table 2. Concepts related to microbial community composition in the rumen of dairy cows, gained using culture-independent methods

\begin{tabular}{ll}
\hline Concept & Reference \\
\hline Species diversity is very large & Kim et al., 2011 \\
A core microbiome is present in all cows & Henderson et al., 2015 \\
Communities may differ across breeds & Bainbridge et al., 2016; Paz et al., 2016 \\
Communities change in response to dietary change & Tajima et al., 2001, Weimer et al., 2010a \\
Communities change in response to age and life stage of individual cows & Rey et al., 2014; Dill-McFarland et al., 2017 \\
Community composition changes during the feeding cycle & Welkie et al., 2010 \\
Communities associated with liquid, solid, and epimural phases differ & Kong et al., 2010 \\
Communities are unique to individual cows and are highly resilient upon perturbation & Weimer et al., 2010b \\
Differences in production metrics (milk yield, milk composition, feed efficiency) & Jami et al., 2014; Jewell et al., 2015; \\
are associated with differences in community composition & Shabat et al., 2016 \\
Community composition results depend in part on methodologies (sampling method, & Henderson et al., 2013 \\
DNA isolation method, sequencing/fingerprinting method used) & \\
\hline
\end{tabular}

a species, making genus-level designations (2 taxonomic levels above strain-level classification) less predictive of metabolic activity. Additionally, $16 \mathrm{~S}$ gene segment sequences do not give a 1:1 ratio of reads per microbial cell, as bacterial species may have as many as 15 copies of the ribosomal DNA operon in their genomes (Acinas et al., 2004). Regardless of the difficulties, ribotyping is still the easiest and most cost-effective means of genotyping microbial populations, and many such surveys have revealed insights into rumen microbial community structure, function, and activity (see Table 2).

Costlier methods attempt to assess microbial metabolic potential directly by sequencing and assembling DNA or RNA sequence reads, thereby circumventing problems with OTU association hypotheses. Improvements to genome assembly algorithms enable the generation of subsections of microbial genomic DNA in large-enough fragments to enable gene prediction and characterization. An even more specialized (and complicated) method involves the sequencing of the metatranscriptome by targeting the RNA fraction of the rumen microbial community. This method directly assesses gene expression, and can therefore provide useful information on metabolic activity more directly. However, because the bulk of microbial gene expression tends to be ribosomal in nature, approximately $90 \%$ of all transcripts typically consist of the $16 \mathrm{~S} / 18 \mathrm{~S}$ and 23S/32S ribosomal subunits (Oliver et al., 2009; Bickhart and Benson, 2011; Chen and Duan, 2011). This redundancy necessitates greater sequencing depth of the transcriptome to capture protein-coding gene transcripts, which in turn increases the costs of such surveys. Additionally, RNA is easily degraded by ubiquitous, ambient RNases in microbially active environments, potentially contributing to a bias in the detection of transcripts or transcript fragments. Such challenges make the use of this method prohibitive for large-scale microbial population phenotyping.

Statistical methods are frequently used after microbial OTU classification or gene discovery to reduce microbial abundance estimates into comparable and easily understood values. Several indices are used by microbial ecologists to reduce microbial species abundance matrices, including Shannon, Chao, Ace, and Unifrac (Lozupone et al., 2011; Morris et al., 2014). These indices are used to estimate parameters such as " $\alpha$ diversity" (the diversity of microbial populations in a single sample) and " $\beta$ diversity" (the diversity of microbial populations between samples), with some modifications that account for species or strain abundance (e.g., weighted Unifrac) to better predict community structure changes (Lozupone et al., 2011). The ability to condense microbial species OTU assignments into singular values should provide some optimism for using microbial community profiles as a useful phenotype in downstream association studies; however, care should be taken in interpreting how variance in a microbial abundance index correlates with a trait. For example, correlation of raw $\alpha$ diversity values with milk production may be difficult to reproduce and may hide the influence of highly abundant species of microbes related to improved production. Moreover, we must resist the temptation to confuse diversity with functionality; in this regard, Shabat et al. (2016) recently showed that higher feed efficiency in dairy cows was associated with lower species diversity, suggesting that optimizing functionality may result from "fine tuning" the community to reduce the abundance of species that do not contribute to overall community function. Nevertheless, when properly done, strategies that generate a reliable, predictive statistic for assessing microbial influence on dairy cattle production will be necessary for downstream association of microbial content with host genotype.

\section{CATTLE RUMEN MICROBIAL PROFILES MAY BE HERITABLE}

One intriguing facet of rumen biology that has been discovered is that rumen contents revert to previously 
established metabolic profiles quite rapidly after being replaced. When rumen contents were transferred from one cow to another, after emptying $\sim 95 \%$ of the rumen contents of the recipient cow, the recipient's rumen $\mathrm{pH}$ and VFA production returned to its pre-transfer profile within $24 \mathrm{~h}$ (Weimer et al., 2010a). This reversion could be explained by 2 or more hypotheses, including the presence of "seed" microbes ready to reestablish themselves after rumen emptying or by intensive host genetic or physiological selection. Reservoirs of microbial seed inoculants for the rumen have not yet been discovered but may explain this rapid reversion; for example, in the contents exchange studies cited above, a small portion of the rumen content was intentionally left as a seed to test for the ability of the native community to recover following the massive challenge by the donor inoculum. However, it is also possible that seed microbes have colonized the rumen epimural layer and rapidly proliferate after rumen emptying/dosing experiments, although little information exists on the strains of microbes that colonize this region. Because the rumen epimural layer represents a direct interface between the host and microbial colonizers, host immunity may play a role in the selection of microbes that colonize on the surface. Alternatively, cattle salivary buffering and rumen $\mathrm{pH}$ regulation, as well as rates of passage (fluid and solids dilution rates), may be the major driving force of this reversion, accounting for a host physiological selection for microbial proliferation. Either of these possibilities, if true, would suggest that host genetics plays a role in microbial content establishment and maintenance.

Investigation of the possibility that rumen microbial profile may be governed by host genetics has been difficult; however, early results have discovered additive genetic effects for the maintenance of general microbial content. For instance, it appears that rumen microbial communities may vary between breeds of cattle and in cross-bred animals (Bainbridge et al., 2016; Paz et al., 2016). Another recent discovery has been that the ratio of archaea to bacteria in the rumen is likely to be heritable (Roehe et al., 2016). This is important in the context of methane emissions from animals fed medium- and high-concentrate diets, as the ratio of archaea: bacteria was found to be correlated $(\mathrm{r}=0.49 ; P<$ 0.001) with methane emissions in live animals (Wallace et al., 2014). Enteric methane emissions have become a flashpoint for the perceived negative environmental impacts of ruminant agriculture (Pitesky et al., 2009). The microbiology of ruminal methanogenesis and prospects for its mitigation have been the subject of several excellent reviews (Knapp et al., 2014; Patra et al., 2017). Because methane production represents a major sink for reducing equivalents produced during ruminal fermentations, decreasing methanogenesis could provide an opportunity to retain feed energy in the form of reduced, metabolizable fermentation products such as propionate. Of interest to the field of statistical genetics is the fact that phenotyping archaea:bacteria ratios could be done on a larger scale by sampling animal rumen contents at slaughter (Wallace et al., 2014) or via infrared spectroscopy of milk to determine methane emissions as a proxy (Vanlierde et al., 2015). In a field plagued by difficulties in efficiently phenotyping microbial population structure across entire populations, an easily assessed proxy biomarker would provide a very important advancement. Still, there is a high degree of diurnal and seasonal variation in rumen microbial contents (Welkie et al., 2010; Noel et al., 2017), potentially making such one-time profiling of questionable value for long-term assessment of rumen metabolic potential. If a robust causality can be demonstrated between specific methanogenic taxa and elevated levels of methane production across time scales, metagenomic investigations of these taxa may lead to specific strategies to control these taxa at the expense of other methanogens having more benign production characteristics.

\section{LINKING THE HERITABILITY OF PRODUCTION TO MICROBIAL SELECTION AND MAINTENANCE}

A central challenge in rumen microbiology is to develop robust and meaningful linkages of microbiome composition and the functional role of individual species or assemblages of species to production variables such as milk yield, milk composition, and feed efficiency. Several studies have identified particular OTUs that display statistically significant positive or negative correlations with these production parameters (Jami et al., 2014; Jewell et al., 2015; Shabat et al., 2016), but to date, almost no systematic attempts have been made to verify the association of any individual OTU across studies. Even within studies, the difficulty in tracing individual OTUs to their deepest phylogenetic level has confounded attempts to interpret the potential role of these OTUs in observed milk production metrics. For example, Jewell et al. (2015) identified 5 OTUs within the genus Prevotella whose abundance was elevated $(P$ $<0.1$ ) in low-RFI (low residual feed intake; i.e., more efficient) cows than in high-RFI (less efficient) cows, along with 7 Prevotella OTUs that were less abundant in the low-RFI cows (Jewell et al., 2015). However, none of these OTUs could be identified to the species level, and even if they could have been, it would be difficult to posit how each OTU within this genus might specifically affect feed efficiency, due to the famously wide metabolic diversity of the genus and the poorly characterized physiology of individual species within 
the genus. Comparisons of $16 \mathrm{~S}$ profiles in lactating cows suggest that milk fat production is correlated with the Firmicutes:Bacteroidetes ratio, explaining 50\% of the variance in milk fat production between individuals (Jami et al., 2014). Although it does not explain many of the mechanisms behind the observed fluctuation in milk fat production, this ratio is particularly useful because it is an easily calculated and predictive value that is not subject to difficulties in OTU resolution mentioned above.

Adding further difficulty, it is challenging to isolate additive microbial effects from host genetic effects on milk production traits due to the incomplete biological characterization of the symbiosis between cattle and rumen microbes. Estimates of the heritability of milk production and components from genetic correlations show an $h^{2}$ value of 0.20 for milk weight, fat percent, and protein percent for Holstein, Jersey, and Brown Swiss cattle (AIP Net Merit 2017; https://www.aipl .arsusda.gov/reference/nmcalc-2017.htm). Given that we know the direct contribution of the rumen microbial metabolism to milk fat and protein through VFA and microbial cell protein production, respectively, the question remains whether the heritable portion of milk component production is due to better animal absorption or processing of these metabolites or due to host selection for specific microbial occupants at key time points in rumen development. A large proportion of the phenotypic variance of milk fat percentage has been attributed to bovine alleles of the diacylglycerol o-acyltransferase 1 (DGAT1) gene (Grisart et al., 2004; Schennink et al., 2007), suggesting that there is a substantial influence on production from the host metabolic side. In the case of $D G A T 1$, the $232 \mathrm{~K}$ allele has a $\mathrm{V}_{\max }$ (the maximum rate of reaction for an enzyme), which changes the proportion of different phospholipids in the mammary epithelial cells (Schennink et al., 2007). Indeed, the amount of variance predicted by this allele of DGAT1 is so strong as to represent a significant marker in genome-wide surveys related to microbial $16 \mathrm{~S}$ profile prediction $[\mathrm{O}$. Gonzalez-Recio (Instituto Nacional de Investigación y Tecnología Agraria y Alimentaria, Madrid, Spain), I. Zubiria (NEIKER-Tecnalia, Vitoria-Gasteiz, Spain), A. Garcia-Rodriguez (NEIKER-Tecnalia, Vitoria-Gasteiz, Spain), A. Hurtado (NEIKER-Tecnalia, Derio, Spain), and R. Atxaerandio (NEIKER-Tecnalia, VitoriaGasteiz, Spain, unpublished data)]. Additional factors that may influence milk fat production may include enzymes related to VFA absorption or capture in the rumen epithelial layer, microbial population selection by the host immune system, and perturbations to metabolic pathways within the liver or mammary cell populations. Given the large range of interacting systems and components and the undoubted influence of management and nutrition on production, it may be difficult to identify all of the influential host genetic factors from such well-used methods as genome-wide association studies, where smaller effects on production are often indistinguishable from background noise. A preferable strategy may be to develop a cost-effective and standardized method for phenotyping microbial populations in the rumen and use it in a downstream model to predict milk production. In the future, we may be focusing on genetic $\times$ environmental $\times$ microbial interactions to explain the variance of milk production in dairy cattle. Such statistical associations - which are very useful from a practical standpoint-could then serve as a springboard for more basic studies that identify the mechanisms through which these interactions operate.

Incidental observations provide hope that more traditional experiments may inform the effects of microbial profile on dairy production. Milk fat depression (MFD) is characterized by a distinctive shift in rumen microbial composition, with Megasphaera eldensii strains increasing in proportion relative to those in non-MFD cows (Weimer et al., 2010b). Interestingly, it is often multiparous cows that break records for per-lactation milk production by a single animal. This is due, in part, to the fact that primiparous cows devote substantial resources to reaching mature BW (Poncheki et al., 2015). However, rumen microbial communities continue to change with maturity (Jewell et al., 2015), and these changes may also play a role in production gains. If such is the case, incidental microbial colonization later in an animal's life could contribute to later gains in production. To better characterize this association, studies may need to focus on time-course microbial sampling within individual animals, as was shown recently (DillMcFarland et al., 2017). If such comparisons can be drawn out over several lactations for a substantial number of animals, the estimation of direct effects of microbial community composition on milk production becomes a distinct possibility. However, the phenotyping of microbial populations in the rumen for statistical modeling is still an unrefined process and is subject to the limitations described above. In order for these studies to succeed, many more surveys of rumen microbial contents using more refined methods are needed.

\section{POSSIBILITIES OF PERTURBING THE MICROBIAL COMMUNITY TO IMPROVE PRODUCTION}

Surveys of rumen gene expression currently point toward a model where the host selects for an initial set of inoculant bacteria or archaea until a core community is established, which is then self-sufficient enough to "police" itself. Expression of toll-like receptors (TLR) and 
cytokines is higher in calves and decreases as animals age (Malmuthuge et al., 2012). Toll-like receptors are cell surface membrane receptors that recognize microbially derived structural molecules and elicit innate immune responses (Akira and Takeda, 2004). Conversely, $\beta$-defensin expression increases with age (Malmuthuge et al., 2012), which suggests that the targeted immune response provided by TLRs and cytokines is eventually replaced by a more passive defense mechanism. Calgranulin expression is also higher in the mature rumen than in other organs, suggesting the suppression of proinflammatory responses as animals age (Xiang et al., 2016). Unlike the large intestine mucosal surface, the rumen expression and immunological profile is more akin to the expression profile of skin epidermis than a gastrointestinal tract membrane (Xiang et al., 2016).
If cattle rumen gene expression experiments provide any insight, the crucial inoculation period appears to be in preweaned calves (Yáñez-Ruiz et al., 2015), right before the transition to a "passive" immune response to rumen contents (see Figure 1). However, this does not rule out the possibility that different feed types may permanently alter rumen microbial content if continuously fed over a long period. Indeed, a recent survey of microbial succession profiles in the feces of dairy cows taken from 2 wk old to first lactation revealed that microbial sequence diversity was low in preweaned calves, but increased quite rapidly after weaning and coincided with cohabitation of calves (Dill-McFarland et al., 2017). This may be due to a strong selection by the host, as evidenced by the expression of TLRs and inflammatory compounds in calves (Malmuthuge et al.,
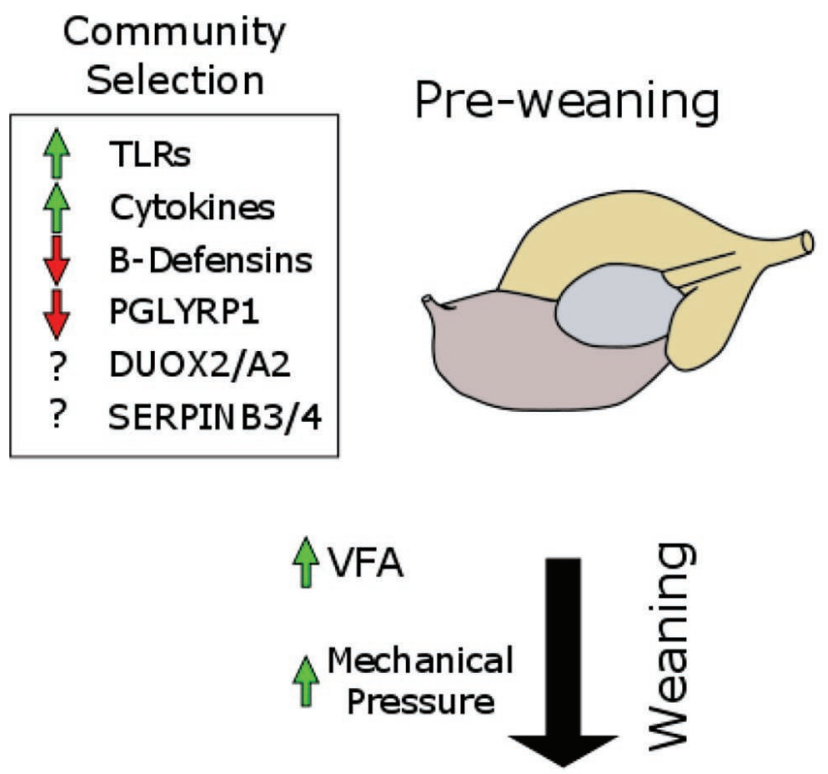

\section{Community Established}

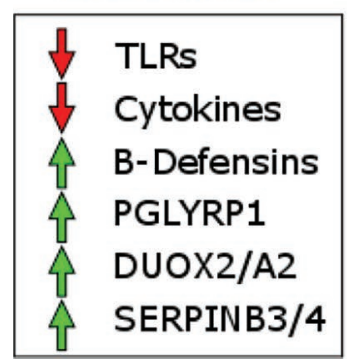

\section{Maturity}

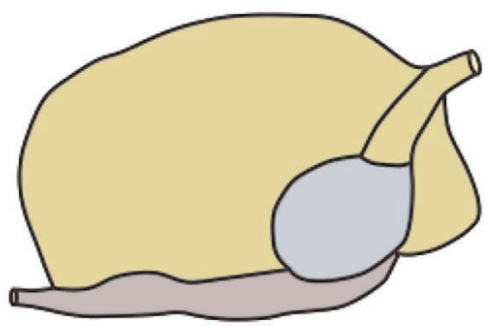

Figure 1. Diagram of rumen epimural expression profile changes. The reticulorumen (dark gray/tan), omasum (mid gray), and abomasum (light gray/brown) major developmental profiles are shown in a preweaning (top) and mature (bottom) state. Relative changes in gene expression are depicted by up/green arrows (increased relative expression) and down/red arrows (decreased relative expression) at each time point. The transition from preweaning to weaned adult animals is indicated by a large down/black arrow, with stimulants for rumen development indicated by small up/green arrows (VFA absorption and mechanical pressure from digesting forage). The changes in gene expression profile from preweaning to maturity suggest a transition from active immune selection for microbes to passive tolerance of their presence. Color version available online. 
2012), or it may indicate a relative "open" period in which external intervention could shape the developing rumen microbial community.

The resiliency of the rumen suggests that-apart from major diet changes (Tajima et al., 2001) - probiotic therapies involving single non-native strains designed to improve production are unlikely to elicit permanent changes in the mature rumen. Indeed, even dosing experiments involving common rumen microbes (Varel et al., 1995; Weimer, 2015) or whole rumen contents (Weimer et al., 2010a) derived from unrelated cows have resulted in only temporary changes in microbial profile or production. Permanent changes are more likely to result from inoculation of microbial species that fill unoccupied niches in the general rumen metabolic landscape (Weimer, 2015), as was found in conferring mimosine resistance to other ruminants upon dosing rumen contents from goats naturally resistant to mimosine due to an established population of the bacterium Synergistes jonesii (Hammond, 1995). A notable (albeit non-ruminal) exception has been the use of probiotic therapies involving mixtures of different bacterial strains to exclude pathogenic Salmonella enterica in poultry (Nisbet et al., 1998; Nisbet, 2002). A mixture of commensal microbes may provide sufficient metabolic sustainability to enable permanent colonization where a single inoculant is unable to sustain itself. Alternatively, there is the possibility of using genetic engineering to modify the metabolic potential of already colonized microbial strains in vivo. By incorporating novel metabolic genes on an existing bacterial plasmid or phage sequence (Simon et al., 1983), it may be possible to introduce new gene content to rumen microbes that have already adapted to the rumen environment. These methods could bypass the aforementioned difficulties of transient probiotic therapies by adapting existing host genomes within the rumen; however, the maintenance of this exogenous DNA within rumen microbial species requires selective pressure. In an attempt to mitigate fluoroacetate poisoning in Australian sheep grazing certain toxic forages, Gregg et al. (1998) successfully established a recombinant strain of Butyrivibrio engineered to contain a dehalogenase gene from a soil $\mathrm{Mo}$ raxella species (Gregg et al., 1998). Protection, though incomplete, was associated with retention of the strain in the subject animals, probably due to the filling of an open metabolic niche. In contrast, a strain of the gut bacterium Bacteroides thetaiotaomicron, engineered to contain an active xylanase from the rumen bacterium Butyrivibrio fibrisolvens, demonstrated persistence during in vitro rumen fermentations only upon use of impractical selection pressure (inclusion of the ancillary substrate chondroitin sulfate); on this basis, its ability to persist in the rumen without artificial supplements was considered unlikely (Cotta et al., 1997). Given the paucity of information we have on rumen bacterial plasmid and phage moieties, along with the well-known difficulty of introducing heterologous genes into various model species of rumen bacteria, this approach will require far better knowledge of the sequence of such constructs.

\section{OTHER CONSIDERATIONS AND FUTURE DIRECTIONS}

The major impediments to rumen microbial functional assessment involve microbial content classification and correlation with host animal production traits, either through the generation of reliable microbial phenotypes or through cross-study comparisons. To date, rumen microbial classification and OTU assignment are typically done on a per-study basis, with only topical comparisons to previous survey results. This presents a substantial bias in interpretation of these results, as the definition of a particular OTU may differ between studies. This can be due to the use of different methods of DNA isolation from rumen contents (Henderson et al., 2013), the use of different OTU variant databases between studies, or to the methods of $16 \mathrm{~S}$ variable region alignment used to classify OTU-specific variation (Nguyen et al., 2016). There is a real need for a comprehensive meta-analysis of all published $16 \mathrm{~S}$ data sets to provide true context to previous discoveries. Much like in eukaryotic whole-genome sequencing surveys, combining all discovered data sets into a larger survey enables better detection of false-positive variants and better detection of strain- or species-specific variant sites. Without these detailed comparisons, each survey will remain "an island" in terms of its ability to be compared against its predecessors and successors. Additionally, methods used to sample microbial contents of the rumen are either too invasive (e.g., cannulae) or require dedicated labor (e.g., stomach tubing), making them infeasible for routine phenotyping. Alternative suggestions for efficiently sampling rumen contents at slaughter (Roehe et al., 2016) may miss key fluctuations in microbial abundance due to feed, stress, and pregnancy, but might provide a suitable baseline for future comparisons. Alternatively, proxy biomarkers or sampling sites may provide a means for frequently and efficiently measuring rumen community composition, as previously mentioned. Because ruminants frequently regurgitate rumen solids for mastication (i.e., chewing the cud), buccal swabs may represent a less laborious method of assessing rumen microbial communities. However, comparisons of buccal microbial community $16 \mathrm{~S}$ profiles with the rumen liquid-phase community in sheep only showed modest $(\mathrm{r} \sim 0.28)$ correlation (Kit- 
telmann et al., 2015). This comparison was likely hampered by the coincidental sampling of the resident oral microbial community in addition to transient rumen microbial species regurgitated from the rumen. Still, it is possible that buccal microbial profiles could be assessed for correlation or covariance with other cattle phenotypic traits or genetic markers, regardless of their predictive potential for individual rumen microbial species.

Whole-genome sequencing may be necessary to tease out the presence and absence of microbial species at a sufficiently high resolution for generating accurate phenotypes. This will require the generation of a host of genomics resources for the constitutive microbial colonizers of the rumen parallel to those available to cattle genetics researchers. Given the paucity of rumen microbial reference genomes, the most useful first step would be the assembly of accurate reference genomes for these species. With these reference genomes, several computational tools become available for rapid classification of whole-genome sequence reads, including read alignment and rapid OTU assignment (Ondov et al., 2016). Given the complexity and variability of the rumen microbial community, a database of microbial reference genomes will need to be constantly updated to include the latest discoveries or pathogens. Recent work done by the Hungate 1000 consortium (http://genome .jgi.doe.gov/TheHunmicrobiome/TheHunmicrobiome .info.html) presents a solid foundation for this effort; however, the species represented in the collection were all cultivable rumen microbial species. Improved methods for sequencing rumen contents in situ and assembling high-quality reference genome assemblies for rumen microbial species from mixed pools will need to be developed to assess the genome sequence of uncultivable species that likely comprise $90 \%$ or more of the rumen microbial community. Once a sufficient number of microbial species have been sequenced and can be reliably genotyped, the potential exists for full metabolic modeling of individual rumen states or high-resolution association analyses. This may identify uninhabited metabolic niches that could be occupied by probiotic microbial species or it may highlight system inefficiencies for correction.

The rumen represents a major evolutionary adaptation by cattle to a diet of highly fibrous forage that is otherwise difficult for nonruminant species to digest. This organ serves as a direct interface between a core group of symbiotic microbes and the host animal and is a key facet of dairy animal nutrition. To better understand the genetic and environmental effects that influence dairy cattle production, it will be necessary to characterize the constituent microbes of a variety of rumens (both healthy and unhealthy, and of high or low efficiency) and to identify the system inputs that affect changes or differences between these states. In the future, we can expect to see genetic selection for animals that select optimal rumen microbial colonizing microbes as part of their natural development. Insights from such studies are likely necessary to permit development of complex probiotic therapies that optimize nutrient production by rumen microbial community.

\section{ACKNOWLEDGMENTS}

The authors were supported by appropriated project 5090-31000-024-00-D, "Determining influence of microbial, feed and animal factors on efficiency of nutrient utilization and performance in lactating dairy cows," of the Agricultural Research Service of the USDA (Washington, DC). Mention of trade names or commercial products in this article is solely for the purpose of providing specific information and does not imply recommendation by the US Department of Agriculture.

\section{REFERENCES}

Acinas, S. G., L. A. Marcelino, V. Klepac-Ceraj, and M. F. Polz. 2004. Divergence and redundancy of $16 \mathrm{~S}$ rRNA sequences in genomes with multiple rrn operons. J. Bacteriol. 186:2629-2635.

Akira, S., and K. Takeda. 2004. Toll-like receptor signalling. Nat. Rev. Immunol. 4:499-511. https://doi.org/10.1038/nri1391.

Bainbridge, M. L., L. M. Cersosimo, A.-D. G. Wright, and J. Kraft. 2016. Rumen bacterial communities shift across a lactation in Holstein, Jersey and Holstein $\times$ Jersey dairy cows and correlate to rumen function, bacterial fatty acid composition and production parameters. FEMS Microbiol. Ecol. 92:fiw059. https://doi.org/10 .1093/femsec/fiw059.

Baldwin, R. L., K. R. McLeod, J. L. Klotz, and R. N. Heitmann. 2004 Rumen development, intestinal growth and hepatic metabolism in the pre- and postweaning ruminant. J. Dairy Sci. 87:E55-E65. https://doi.org/10.3168/jds.S0022-0302(04)70061-2.

Bergman, E. N. 1990. Energy contributions of volatile fatty acids from the gastrointestinal tract in various species. Physiol. Rev. $70: 567-590$.

Bickhart, D. M., and D. R. Benson. 2011. Transcriptomes of Frankia sp. strain CcI3 in growth transitions. BMC Microbiol. 11:192. https://doi.org/10.1186/1471-2180-11-192.

Chen, Z., and X. Duan. 2011. Ribosomal RNA depletion for massively parallel bacterial RNA-sequencing applications. Methods Mol. Biol. 733:93-103. https://doi.org/10.1007/978-1-61779-089-8_7.

Cotta, M. A., T. R. Whitehead, and M. A. Rasmussen. 1997. Survival of the recombinant Bacteroides thetaiotaomicron strain BTX in in vitro rumen incubations. J. Appl. Microbiol. 82:743-750. https:// doi.org/10.1046/j.1365-2672.1997.00152.x

Dill-McFarland, K. A., J. D. Breaker, and G. Suen. 2017. Microbial succession in the gastrointestinal tract of dairy cows from 2 weeks to first lactation. Sci. Rep. 7:40864. https://doi.org/10.1038/ srep40864.

Gregg, K., B. Hamdorf, K. Henderson, J. Kopecny, and C. Wong. 1998. Genetically modified ruminal bacteria protect sheep from fluoroacetate poisoning. Appl. Environ. Microbiol. 64:3496-3498.

Grisart, B., F. Farnir, L. Karim, N. Cambisano, J.-J. Kim, A. Kvasz, M. Mni, P. Simon, J.-M. Frère, W. Coppieters, and M. Georges. 2004. Genetic and functional confirmation of the causality of the DGAT1 K232A quantitative trait nucleotide in affecting milk yield and composition. Proc. Natl. Acad. Sci. USA 101:2398-2403. 
Hammond, A. C. 1995. Leucaena toxicosis and its control in ruminants. J. Anim. Sci. 73:1487-1492.

Henderson, G., F. Cox, S. Ganesh, A. Jonker, and W. Young., Global Rumen Census Collaborators, andJanssen, P. H. 2015. Rumen microbial community composition varies with diet and host, but a core microbiome is found across a wide geographical range. Sci. Rep. 5:14567. https://doi.org/10.1038/srep14567.

Henderson, G., F. Cox, S. Kittelmann, V. H. Miri, M. Zethof, S. J. Noel, G. C. Waghorn, and P. H. Janssen. 2013. Effect of DNA extraction methods and sampling techniques on the apparent structure of cow and sheep rumen microbial communities. PLoS One 8:e74787. https://doi.org/10.1371/journal.pone.0074787.

Jami, E., B. A. White, and I. Mizrahi. 2014. Potential role of the bovine rumen microbiome in modulating milk composition and feed efficiency. PLoS One 9:e85423. https://doi.org/10.1371/journal .pone.0085423.

Jewell, K. A., C. A. McCormick, C. L. Odt, P. J. Weimer, and G. Suen. 2015. Ruminal bacterial community composition in dairy cows is dynamic over the course of two lactations and correlates with feed efficiency. Appl. Environ. Microbiol. 81:4697-4710. https://doi.org/10.1128/AEM.00720-15.

Kim, M., M. Morrison, and Z. Yu. 2011. Status of the phylogenetic diversity census of ruminal microbiomes. FEMS Microbiol. Ecol. 76:49-63. https://doi.org/10.1111/j.1574-6941.2010.01029.x.

Kittelmann, S., M. R. Kirk, A. Jonker, A. McCulloch, and P. H. Janssen. 2015. Buccal swabbing as a noninvasive method to determine bacterial, archaeal, and eukaryotic microbial community structures in the rumen. Appl. Environ. Microbiol. 81:7470-7483. https://doi .org/10.1128/AEM.02385-15.

Knapp, J. R., G. L. Laur, P. A. Vadas, W. P. Weiss, and J. M. Tricarico. 2014. Invited review: Enteric methane in dairy cattle production: Quantifying the opportunities and impact of reducing emissions. J. Dairy Sci. 97:3231-3261. https://doi.org/10.3168/jds 2013-7234.

Kong, Y., R. Teather, and R. Forster. 2010. Composition, spatial distribution, and diversity of the bacterial communities in the rumen of cows fed different forages. FEMS Microbiol. Ecol. 74:612-622. https://doi.org/10.1111/j.1574-6941.2010.00977.x.

Lozupone, C., M. E. Lladser, D. Knights, J. Stombaugh, and R. Knight. 2011. UniFrac: An effective distance metric for microbial community comparison. ISME J. 5:169-172. https://doi.org/10 .1038 /ismej.2010.133.

Malmuthuge, N., M. Li, P. Fries, P. J. Griebel, and L. L. Guan. 2012. Regional and age dependent changes in gene expression of Tolllike receptors and key antimicrobial defence molecules throughout the gastrointestinal tract of dairy calves. Vet. Immunol. Immunopathol. 146:18-26. https://doi.org/10.1016/j.vetimm.2012.01.010.

Morris, E. K., T. Caruso, F. Buscot, M. Fischer, C. Hancock, T. S. Maier, T. Meiners, C. Müller, E. Obermaier, D. Prati, S. A. Socher, I. Sonnemann, N. Wäschke, T. Wubet, S. Wurst, and M. C. Rillig. 2014. Choosing and using diversity indices: Insights for ecological applications from the German Biodiversity Exploratories. Ecol. Evol. 4:3514-3524. https://doi.org/10.1002/ece3.1155.

Nguyen, N.-P., T. Warnow, M. Pop, and B. White. 2016. A perspective on $16 \mathrm{~S}$ rRNA operational taxonomic unit clustering using sequence similarity. NPJ Biofilms Microbiomes 2:16004. https://doi .org/10.1038/npjbiofilms.2016.4.

Nisbet, D. 2002. Defined competitive exclusion cultures in the prevention of enteropathogen colonisation in poultry and swine. Antonie van Leeuwenhoek 81:481. https://doi.org/10.1023/A: 1020541603877.

Nisbet, D. J., G. I. Tellez, V. K. Lowry, R. C. Anderson, G. Garcia, G. Nava, M. H. Kogut, D. E. Corrier, and L. H. Stanker. 1998. Effect of a commercial competitive exclusion culture (Preempt) on mortality and horizontal transmission of Salmonella gallinarum in broiler chickens. Avian Dis. 42:651-656.

Noel, S. J., G. T. Attwood, J. Rakonjac, C. D. Moon, G. C. Waghorn, and P. H. Janssen. 2017. Seasonal changes in the digesta-adherent rumen bacterial communities of dairy cattle grazing pasture. PLoS One 12:e0173819. https://doi.org/10.1371/journal.pone.0173819.
Oliver, H. F., R. H. Orsi, L. Ponnala, U. Keich, W. Wang, Q. Sun, S. W. Cartinhour, M. J. Filiatrault, M. Wiedmann, and K. J. Boor. 2009. Deep RNA sequencing of $L$. monocytogenes reveals overlapping and extensive stationary phase and sigma B-dependent transcriptomes, including multiple highly transcribed noncoding RNAs. BMC Genomics 10:641. https://doi.org/10.1186/1471-2164 -10-641.

Ondov, B. D., T. J. Treangen, P. Melsted, A. B. Mallonee, N. H. Bergman, S. Koren, and A. M. Phillippy. 2016. Mash: Fast genome and metagenome distance estimation using MinHash. Genome Biol. 17:132. https://doi.org/10.1186/s13059-016-0997-x.

Patra, A., T. Park, M. Kim, and Z. Yu. 2017. Rumen methanogens and mitigation of methane emission by anti-methanogenic compounds and substances. J. Anim. Sci. Biotechnol. 8:13. https://doi .org/10.1186/s40104-017-0145-9.

Paz, H. A., C. L. Anderson, M. J. Muller, P. J. Kononoff, and S. C. Fernando. 2016. Rumen bacterial community composition in Holstein and Jersey cows is different under same dietary condition and is not affected by sampling method. Front. Microbiol. 7:1206. https://doi.org/10.3389/fmicb.2016.01206.

Pitesky, M. E., K. R. Stackhouse, and F. M. Mitloehner. 2009. Clearing the air. Adv. Agron. 103:1-40. https://doi.org/10.1016/S0065 -2113(09)03001-6.

Poncheki, J. K., M. L. S. Canha, S. L. Viechnieski, R. de Almeida, J. K. Poncheki, M. L. S. Canha, S. L. Viechnieski, and R. de Almeida. 2015. Analysis of daily body weight of dairy cows in early lactation and associations with productive and reproductive performance. Rev. Bras. Zootec. 44:187-192. https://doi.org/10.1590/ S1806-92902015000500004.

Poulsen, M., C. Schwab, B. B. Jensen, R. M. Engberg, A. Spang, N. Canibe, O. Højberg, G. Milinovich, L. Fragner, C. Schleper, W. Weckwerth, P. Lund, A. Schramm, and T. Urich. 2013. Methylotrophic methanogenic Thermoplasmata implicated in reduced methane emissions from bovine rumen. Nat. Commun. 4:1428. https://doi.org/10.1038/ncomms2432.

Rey, M., F. Enjalbert, S. Combes, L. Cauquil, O. Bouchez, and V. Monteils. 2014. Establishment of ruminal bacterial community in dairy calves from birth to weaning is sequential. J. Appl. Microbiol. 116:245-257. https://doi.org/10.1111/jam.12405.

Roehe, R., R. J. Dewhurst, C.-A. Duthie, J. A. Rooke, N. McKain, D. W. Ross, J. J. Hyslop, A. Waterhouse, T. C. Freeman, M. Watson, and R. J. Wallace. 2016. Bovine host genetic variation influences rumen microbial methane production with best selection criterion for low methane emitting and efficiently feed converting hosts based on metagenomic gene abundance. PLoS Genet. 12:e1005846. https://doi.org/10.1371/journal.pgen.1005846.

Schennink, A., W. M. Stoop, M. H. P. W. Visker, J. M. L. Heck, H. Bovenhuis, J. J. van der Poel, H. J. F. van Valenberg, and J. A. M. van Arendonk. 2007. DGAT1 underlies large genetic variation in milk-fat composition of dairy cows. Anim. Genet. 38:467-473. https://doi.org/10.1111/j.1365-2052.2007.01635.x.

Shabat, S. K. B., G. Sasson, A. Doron-Faigenboim, T. Durman, S. Yaacoby, M. E. Berg Miller, B. A. White, N. Shterzer, and I. Mizrahi. 2016. Specific microbiome-dependent mechanisms underlie the energy harvest efficiency of ruminants. ISME J. 10:2958-2972. https://doi.org/10.1038/ismej.2016.62.

Simon, R., U. Priefer, and A. Pühler. 1983. A broad host range mobilization system for in vivo genetic engineering: Transposon mutagenesis in gram negative bacteria. Nat. Biotechnol. 1:784-791. https://doi.org/10.1038/nbt1183-784.

Tajima, K., R. I. Aminov, T. Nagamine, H. Matsui, M. Nakamura, and Y. Benno. 2001. Diet-dependent shifts in the bacterial population of the rumen revealed with real-time PCR. Appl. Environ. Microbiol. 67:2766-2774. https://doi.org/10.1128/AEM.67.6.2766 $-2774.2001$.

Vanlierde, A., M.-L. Vanrobays, F. Dehareng, E. Froidmont, H. Soyeurt, S. McParland, E. Lewis, M. H. Deighton, F. Grandl, M. Kreuzer, B. Gredler, P. Dardenne, and N. Gengler. 2015. Hot topic: Innovative lactation-stage-dependent prediction of methane emissions from milk mid-infrared spectra. J. Dairy Sci. 98:57405747. https://doi.org/10.3168/jds.2014-8436. 
Varel, V. H., J. T. Yen, and K. K. Kreikemeier. 1995. Addition of cellulolytic clostridia to the bovine rumen and pig intestinal tract. Appl. Environ. Microbiol. 61:1116-1119.

Wallace, R. J., J. A. Rooke, C.-A. Duthie, J. J. Hyslop, D. W. Ross, N. McKain, S. M. de Souza, T. J. Snelling, A. Waterhouse, and R. Roehe. 2014. Archaeal abundance in post-mortem ruminal digesta may help predict methane emissions from beef cattle. Sci. Rep. 4:5892. https://doi.org/10.1038/srep05892.

Weimer, P. J. 2015. Redundancy, resilience, and host specificity of the ruminal microbiota: implications for engineering improved ruminal fermentations. Front. Microbiol. 6:296. https://doi.org/10.3389/ fmicb.2015.00296.

Weimer, P. J., D. M. Stevenson, H. C. Mantovani, and S. L. C. Man. 2010a. Host specificity of the ruminal bacterial community in the dairy cow following near-total exchange of ruminal contents1. J. Dairy Sci. 93:5902-5912. https://doi.org/10.3168/jds.2010-3500.

Weimer, P. J., D. M. Stevenson, and D. R. Mertens. 2010b. Shifts in bacterial community composition in the rumen of lactating dairy cows under milk fat-depressing conditions. J. Dairy Sci. 93:265278. https://doi.org/10.3168/jds.2009-2206.
Welkie, D. G., D. M. Stevenson, and P. J. Weimer. 2010. ARISA analysis of ruminal bacterial community dynamics in lactating dairy cows during the feeding cycle. Anaerobe 16:94-100. https://doi .org/10.1016/j.anaerobe.2009.07.002.

Xiang, R., V. H. Oddy, A. L. Archibald, P. E. Vercoe, and B. P. Dalrymple. 2016. Epithelial, metabolic and innate immunity transcriptomic signatures differentiating the rumen from other sheep and mammalian gastrointestinal tract tissues. PeerJ 4:e1762. https://doi.org/10.7717/peerj.1762.

Yáñez-Ruiz, D. R., L. Abecia, and C. J. Newbold. 2015. Manipulating rumen microbiome and fermentation through interventions during early life: A review. Front. Microbiol. 6:1133. https://doi.org/10 $.3389 /$ fmicb.2015.01133.

Yang, W. Z., K. A. Beauchemin, and L. M. Rode. 2001. Effect of dietary factors on distribution and chemical composition of liquid- or solid-associated bacterial populations in the rumen of dairy cows. J. Anim. Sci. 79:2736-2746. 